\title{
BreastCore
}

\section{The Role of CDK 4/6 Inhibitors in Breast Cancer Treatment}

\author{
Isabell Witzel Volkmar Müller \\ Clinic for Gynecology and Obstetrics, University Medical Center Hamburg, Hamburg, Germany
}

Almost two thirds of breast cancers express the estrogen receptor (ER), which plays an important role in malignant growth stimulation and as therapeutic target. In postmenopausal patients, aromatase inhibitors (AI, e.g., letrozole and anastrozole) have become the treatment of choice in first-line therapy [1]. Unfortunately, some patients have a primary or de novo resistance to first-line endocrine therapy and even patients who initially respond will acquire resistance to treatment and relapse. On disease progression, second-line treatment options include other classes of AI (steroidal or nonsteroidal) and the ER antagonists fulvestrant and tamoxifen [2].

The study of resistance to endocrine therapies in hormone receptor (HR)-positive breast cancer has aimed at identifying new therapeutic strategies that enhance the efficacy of endocrine therapies. However, endocrine resistance is a frequent problem in breast cancer treatment. Yet, insights into estrogen mediated signaling allowed the development of therapeutic approaches interacting with the cell cycle which might overcome endocrine resistance in breast cancer patients.

Dysregulation of the cell-cycle control is a frequent event in breast cancer and occurs via a number of different mechanisms. These dysregulations involving components of CDK4/6 and cyclin D lead to a survival advantage of the cancer cell. CDK4/6 inhibition can reduce cell growth and suppress DNA replication in tumors with functional tumor-suppressor retinoblastoma protein (RB). The cell-cycle machinery is important for efficacy of hormonal therapy in breast cancer, as ER-positive RB-negative xenograft models are resistant to tamoxifen [3].

In ER-positive metastatic breast cancer, often several lines of therapy are effective until patients require chemotherapy. Cellcycle control is a very promising additional option to prolong progression-free survival and time until chemotherapy is needed. Results from the phase III study PALOMA 3 showed that adding palbociclib to fulvestrant more than doubled the duration of disease control. Women with previously treated, HR-positive, HER2negative advanced breast cancer [4] gained almost 5 months of disease control. Palbociclib plus fulvestrant allowed patients to maintain good quality of live (QoL) in the endocrine resistance setting while experiencing substantially delayed disease progression [5]. In the phase II trial PALOMA 1 evaluating palbociclib in combination with letrozole in treatment-naïve patients, an improvement in progression-free survival could be seen (26.1 vs. 7.5 months) [6]. The results of the phase III trial PALOMA 2 evaluating letrozole with or without palbociclib in HR-positive, HER2negative advanced breast cancer patients presented at the ASCO Annual Meeting in June 2016 confirmed these positive findings [7]. All trials illustrate the high potential of CDK4/6 inhibition and the clinical impact of this new treatment approach.

In this issue of BREAST CARE, Marcus Schmidt will highlight pre-clinical data and early clinical trials which led to an accelerated approval of palbociclib by the US Food and Drug Administration (FDA) as first-line treatment in combination with letrozole in advanced HR-positive and HER2-negative breast cancer [8].

Johannes Ettl adds more data about the clinical trials investigating palbociclib [9]. To date, 2 large clinical trials have been fully published and one was presented at a meeting. In his article, he discusses the results of these trials and their clinical relevance for the management of HR-positive advanced breast cancer. In addition, he gives information about QoL measurement in patients treated in the PALOMA 3 trial.

Romualdo Barroso-Sousa and colleagues give an overview about new upcoming components, mainly abemaciclib and ribociclib [10]. The preclinical and clinical data are described as well as toxicity profiles and drug action. Ribociclib has already been investigated in the combination with antiestrogens in the MONALEESA trials and also with additional PI3K inhibitors. Abemaciclib is the only CDK4/6 inhibitor that passes the blood-brain barrier. But it also has a different toxicity profile. The combination with antiestrogens has been investigated in the MONARCH trials.

Selective CDK4/6 inhibitors represent an important therapeutic advance in HR-positive breast cancer. The role in other breast can-

\section{KARGER}

Fax +497614520714
() 2016 S. Karger GmbH, Freiburg

1661-3791/16/0113-0165\$39.50/0
PD Dr. med. Isabell Witzel 
cer subtypes and the combination with other agents will be of further interest. The articles in this issue of BREAST CARE give an overview of the current status and directions for future development that will help to improve treatment of breast cancer patients.

\section{Disclosure Statement}

The authors declare that they have no conflict of interest.

\section{References}

1 Smith IE, Dowsett M: Aromatase inhibitors in breast cancer. N Engl J Med 2003;348:2431-2442.

2 Chia S, Gradishar W, Mauriac L, Bines J, Amant F, Federico M, Fein L, Romieu G, Buzdar A, Robertson JF, Brufsky A, Possinger K, Rennie P, Sapunar F, Lowe E, Piccart M: Double-blind, randomized placebo controlled trial of fulvestrant compared with exemestane after prior nonsteroidal aromatase inhibitor therapy in postmenopausal women with hormone receptor-positive, advanced breast cancer: results from EFECT. J Clin Oncol 2008;26:1664-1670.

3 Bosco EE, Wang Y, Xu H, Zilfou JT, Knudsen KE, Aronow BJ, Lowe SW, Knudsen ES: The retinoblastoma tumor suppressor modifies the therapeutic response of breast cancer. J Clin Invest 2007;117:218-228.

4 Cristofanilli M, Turner NC, Bondarenko I, Ro J, Im SA, Masuda N, Colleoni M, DeMichele A, Loi S, Verma S, Iwata H, Harbeck N, Zhang K, Theall KP, Jiang Y, Bartlett CH, Koehler M, Slamon D: Fulvestrant plus palbociclib versus fulvestrant plus placebo for treatment of hormone-receptor-positive, HER2negative metastatic breast cancer that progressed on previous endocrine therapy (PALOMA-3): final analysis of the multicentre, double-blind, phase 3 randomised controlled trial. Lancet Oncol 2016; DOI: 10.1016/S1470-2045(15)00613-0.
Harbeck N, Iyer S, Turner N, Cristofanilli M, Ro J, Andre F, Loi S, Verma S, Iwata H, Bhattacharyya $\mathrm{H}$, Puyana Theall K, Bartlett CH, Loibl S: Quality of life with palbociclib plus fulvestrant in previously treated hormone receptor-positive, HER2-negative metastatic breast cancer: patient-reported outcomes from the PALOMA-3 trial. Ann Oncol 2016; doi: 10.1093/annonc/mdw139.

6 Finn RS, Crown JP, Lang I, Boer K, Bondarenko IM, Kulyk SO, Ettl J, Patel R, Pinter T, Schmidt M, Shparyk Y, Thummala AR, Voytko NL, Fowst C, Huang X, Kim ST, Randolph S, Slamon DJ: The cyclin-dependent kinase 4/6 inhibitor palbociclib in combination with letrozole versus letrozole alone as first-line treatment of oestrogen receptor-positive, HER2-negative, advanced breast cancer (PALOMA-1/TRIO-18): a randomised phase 2 study. Lancet Oncol 2015;16:25-35.
7 Finn et al. PALOMA-2: Primary results from a phase III trial of palbociclib (P) with letrozole (L) compared with letrozole alone in postmenopausal women with ER+/HER2- advanced breast cancer (ABC). J Clin Oncol 2016;34 (suppl) abstr 507.

8 Schmidt M: Palbociclib - from bench to bedside and beyond. Breast Care 2016; DOI: 10.1159/000447001.

9 Ettl J: Palbociclib: First CDK4/6 Inhibitor in clinical practice for the treatment of advanced HR-positive breast cancer. Breast Care 2016; DOI: 10.1159/ 000447417.

10 Barroso-Sousa R, Shapiro GI, Tolaney SM: Clinical development of the CDK4/6 inhibitors ribociclib and abemaciclib in breast cancer. Breast Care 2016; DOI: $10.1159 / 000447284$. 